\title{
Modeling Rift Valley Fever with Treatment and Trapping Control Strategies
}

\author{
Jonnes Lugoye1, Josephine Wairimu², C. B. Alphonce1, Marilyn Ronoh ${ }^{2}$ \\ ${ }^{1}$ Univeristy of Dar es Salaam, Dar es Salaam, Tanzania \\ ${ }^{2}$ School of Mathematics, University of Nairobi, Nairobi, Kenya \\ Email: lugoye.jonnes@gmail.com, jwndirangu@uonbi.ac.ke, alphonce@maths.udsm.ac.tz, \\ mcronoh1@gmail.com
}

Received 8 December 2015; accepted 27 March 2016; published 30 March 2016

Copyright (C) 2016 by authors and Scientific Research Publishing Inc.

This work is licensed under the Creative Commons Attribution International License (CC BY). http://creativecommons.org/licenses/by/4.0/

(c) O) Open Access

\section{Abstract}

We consider a rift valley fever model with treatment in human and livestock populations and trapping in the vector (mosquito) population. The basic reproduction number $\mathcal{R}_{0}$ is established and used to determine whether the disease dies out or is established in the three populations. When $\mathcal{R}_{0} \leq 1$, the disease-free equilibrium is shown to be globally asymptotically stable and the disease does not spread and when $\mathcal{R}_{0}>1$, a unique endemic equilibrium exists which is globally stable and the disease will spread. The mathematical model is analyzed analytically and numerically to obtain insight of the impact of intervention in reducing the burden of rift valley fever disease's spread or epidemic and also to determine factors influencing the outcome of the epidemic. Sensitivity analysis for key parameters is also done.

\section{Keywords}

\section{Rift Valley Fever, Mosquito Trapping, Treatment, Rift Valley Fever Control}

\section{Introduction}

Rift Valley Fever (RVF) is an infectious disease caused by the RVF virus of the genus Phlebovirus and family Bunyaviridae. It is transmitted between animal species, including cattle, sheep, goats, and camels, primarily through the bite of the female mosquito, usually Aedes or Culex [1]. Gaff [2] formulated an epidemiological model of RVFV determining how to reduce egg classes of mosquitoes. Clements [3] modeled the distribution of two species of mosquitoes (Aedes aegypti and Culex pipiens complex) and showed that distribution of vectors had biological and epidemiological significance in relation to disease outbreak hotspots, and provided guidance for the selection of sampling areas for RVF vectors during inter-epidemic periods. Fischer in [4] studied the transmission potential of Rift Valley Fever virus in Netherlands by developing a mathematical model to deter- 
mine the initial growth and Floquet ratios which were indicators of the probability of an outbreak and persistence in a periodic changing environment caused by seasonality. Their result showed that several areas of Netherlands had a high transmission potential and risk persistence of the infection [2]. The key result is that RVF virus can persist in a closed system for 10 years if the contact rate between hosts and vectors is high [5]. Meshe [6] formulated and analysed a mathematical model described by a system of non-linear ordinary differential equations to gain insight on the dynamics of RVF in mosquito, livestock and human hosts. The disease's threshold was computed and used to investigate the local stability of the equilibria and infer the behaviour of the disease. Tianchan et al. [7] developed a mathematical model incorporating the effect of space into the mathematical model of RVF to study the effect of the virus spread as affected by the movements of livestock, human and mosquitoes. The simulated results showed that different geographic spaces have a great effect on the spread of the pathogen and the disease in general. [8] presented the mathematical model for Rift Valley fever (RVF) transmission in cattle and mosquitoes by extending the existing models for vector-borne diseases to include an asymptomatic host class and vertical transmission in vectors. RVF remains a threat to livestock keepers and nations where the disease is occurring due to its major economic implications through the costs of the measures taken at individual, collective and international levels to prevent or control infections and disease outbreaks [9]. In this study we extend the work of [6] by incorporating the aspect of control in the modelling transmission dynamics of RVF in humans and animals, by answering the question: How does trapping of mosquitoes and/or treatment of humans and animals or both affect the spread of the disease?

The rest of the paper is arranged as follows. In Section 2, we formulate the mathematical model and establish the basic properties of the model. In Section 3, we compute the basic reproduction number herein referred to as the effective reproduction number, and determine the local and global stability of the Disease Free equilibrium. In Section 4, we establish the existence and stability of the Endemic Equilibrium. In Section 5, we have sensitivity analysis with its interpretation. Section 6 has numerical simulation and Section 7 is the conclusion.

\section{Model Formulation}

In this model we divide the three populations into the susceptible, $S_{i}$ and infected, $I_{i}$ classes, for $i=h, l, m$ for, human $(h)$, livestock $(I)$ and mosquitoes $(m)$, respectively. The three susceptible populations become infected via an infectious mosquito bite at per capita rates $\lambda_{i j}$. The newborns in each category are recruited at the per capita birth rate of $\pi_{i}$ and hosts either die naturally or owing to the disease at per capita rates $\mu_{i}$ and $\delta_{i}$, respectively. Treatment in livestock is introduced at a constant rate $\gamma_{l}$; treatment in humans at a constant rate $\gamma_{h}$ and trapping in mosquito at a constant rate $\gamma_{m}$ resulting in the classes of treated livestock $T_{l}$, treated humans $T_{h}$ and trapped mosquito $T_{p}$. We assume that treated human and livestock recover at a constant rate $\eta_{h}, \eta_{l}$ respectively and return to the susceptible class again. The susceptible vector is trapped at a constant rate $\sigma_{h}$. Since a population dynamics model is considered, all the state variables and parameters are assumed to be non-negative, with as SI framework. The model assumes that individuals mix homogeneously in the human and livestock population where all individuals have equal chance of getting the infection if they come into contact with infectious mosquitoes and that transmission of the infection occurs with a standard incidence. It is the assumption of the model that there is natural mortality and disease induced death for livestock and human beings, whereas mosquitoes die only naturally, thus there is no disease induced death for mosquitoes. Again the model assumes that the individuals infected with rift valley from all three populations do not recover naturally. The schematic diagram is given below

$$
\left\{\begin{array}{l}
S_{h}^{\prime}=\pi_{h}-\lambda_{h m} S_{h}-\left(\mu_{h}+\delta_{h}\right) S_{h}+\eta_{h} T_{h} \\
I_{h}^{\prime}=\lambda_{h m} S_{h}-\left(\mu_{h}+\delta_{h}+\gamma_{h}\right) I_{h}, \\
T_{h}^{\prime}=\gamma_{h} I_{h}-\left(\mu_{h}+\eta_{h}+\delta_{h}\right) T_{h}, \\
S_{l}^{\prime}=\pi_{l}-\lambda_{l m} S_{l}-\mu_{l} S_{l}+\eta_{l} T_{l}, \\
I_{l}^{\prime}=\lambda_{l m} S_{l}-\left(\mu_{l}+\delta_{l}+\gamma_{l}\right) I_{l}, \\
T_{l}^{\prime}=\gamma_{l} I_{l}-\left(\eta_{l}+\delta_{l}+\mu_{l}\right) T_{l}, \\
S_{m}^{\prime}=\pi_{m}-\left(\lambda_{m l}+\lambda_{m h}\right) S_{m}-\left(\mu_{m}+\sigma_{m}\right) S_{m} \\
I_{m}^{\prime}=\left(\lambda_{m l}+\lambda_{m h}\right) S_{m}-\left(\mu_{m}+\gamma_{m}\right) I_{m}, \\
R_{h}^{\prime}=\rho T_{h}-\left(\mu_{h}+\delta_{h}\right) R_{h}, \\
T_{p}^{\prime}=\sigma_{m} S_{m}+\gamma_{m} I_{m} .
\end{array}\right.
$$


with initial conditions, $\left(S_{h}(0), I_{h}(0), T_{h}(0), S_{l}(0), I_{l}(0), T_{l}(0), S_{m}(0), I_{m}(0), T_{p}(0)\right)$. The force of infections are given by $\lambda_{h m}=\frac{\beta_{1} I_{m}}{N_{h}}, \lambda_{l m}=\frac{\beta_{2} I_{m}}{N_{l}}, \lambda_{m l}=\frac{\beta_{3} I_{l}}{N_{m}}, \lambda_{m h}=\frac{\beta_{4} I_{h}}{N_{m}}$. The parameters, $\beta_{1}, \beta_{2}, \beta_{3}$, and $\beta_{4}$ are the transmission rates. Adding equations system 1 , we have

$$
N_{h}^{\prime}=\pi_{i}-\mu N_{i}-\delta_{i} I_{i} .
$$

\subsection{Model Analysis}

In this section, we carry out stability analysis of the model (1). The model properties are employed to establish criteria for positivity of solutions and well-possessedness of the system.

\subsubsection{Invariant Region}

In this section a region in which solutions of the model system (4.1) are uniformly bounded in a proper subset $\Omega_{1} \in \mathcal{R}_{+}^{10}$. Let $\left(S_{h}, I_{h}, T_{h}, S_{l}, I_{l}, T_{l}, S_{m}, I_{m}, T_{p}\right) \in \mathcal{R}_{+}^{10}$ be any solution with positive initial conditions. Then from Equation (4.1) it is noted that in the absence of the disease (i.e. $\delta=0$ ), the total host population size is given by,

$$
N_{h}^{\prime}=\pi_{i}-\mu N_{i}
$$

so

$$
0 \leq N_{i} \leq \frac{\pi_{i}}{\mu_{i}}+N_{i}(0) \mathrm{e}^{-\mu_{i}} t
$$

where $N_{i}(0)$ is the value evaluated at the initial conditions of the respective variables. Thus, as $t \rightarrow \infty, 0 \leq N_{i} \leq \pi_{i} \rightarrow \infty$. In respect of this, all the feasible solutions of system (1) enter the region

$$
\Omega=\left(S_{h}, I_{h}, R_{h}, T_{h}, S_{l}, I_{l}, T_{l}, S_{m}, I_{m}, T_{p}\right) \in R_{+}^{10}: N_{i} \leq \frac{\pi_{i}}{\mu_{i}}=N_{i}^{*} .
$$

Hence, $\Omega_{1}$ is positively invariant and it is sufficient to consider solutions in $\Omega_{1}$. Furthermore, existence, uniqueness and continuation of results for system 1 hold in this region. It is clear that all solutions of model system (1) starting in $\Omega_{1}^{*}$ remain in $\Omega_{1} \forall t \geq 0$. Since the model monitors populations, all parameters and state variables for system 1 are assumed to be positive. The result is summarized in the following lemma.

\section{Lemma}

The region $\Omega \in \mathcal{R}_{+}^{10}$ is positively invariant for the model system (1) with initial conditions in $\mathcal{R}_{+}$.

\subsubsection{Positivity of Solutions}

\section{Lemma}

Let the initial data be $S_{h}(0), I_{h}(0), T_{h}(0), S_{l}(0), I_{l}(0), T_{l}(0), S_{m}(0), I_{m}(0), T_{p}(0) \in \Omega_{1}$; Then the solution set $S_{h}(t), I_{h}(t), T_{h}(t), S_{l}(t), I_{l}(t), T_{l}(t), S_{m}(t), I_{m}(t), T_{p}(t)$ of the system 1 is positive $\forall t \geq 0$.

Proof From the first equation of the model system 1

$$
S_{h}^{\prime}=\pi_{h}-\lambda_{h m} S_{h}-\mu_{h} S_{h}+\eta_{h} T_{h} \geq-\left(\lambda_{h m}+\mu_{h}\right) S_{h},
$$

that is

$$
S_{h}^{\prime}=-\left(\lambda_{h m}+\mu_{h}\right) S_{h},
$$

integrating by the equation above gives,

$$
\begin{gathered}
\int \frac{\mathrm{d} S_{h}}{S_{h}} \geq \int_{0}^{t}-\left(\lambda_{h m}+\mu_{h}\right) S_{h} \mathrm{~d} t, \\
\ln \left[S_{h}\right]_{0}^{t} \geq-\int_{0}^{t}\left(\lambda_{h m}+\mu_{h}\right) S_{h} \mathrm{~d} t,
\end{gathered}
$$




$$
\ln \left[\frac{S_{h}(t)}{S_{h}(0)}\right] \geq \exp ^{-\int_{0}^{t}\left(\lambda_{h m}+\mu_{h}\right) S_{h} \mathrm{dt}}
$$

Then

$$
S_{h}(t) \geq S_{h}(0) \exp ^{-\int_{0}^{t}\left(\lambda_{h m}+\mu_{h}\right) S_{h} \mathrm{dt}}>0 .
$$

Similarly, it can be shown that the remaining eight equations of system (4.1) are also positive $\forall t>0$.

\section{Steady State Solutions}

In this section the model system (4.1) is qualitatively analysed by determining the equilibria, carrying out their corresponding stability analysis and interpreting the results. Let $E=\left(S_{h}^{*}, I_{h}^{*}, T_{h}^{*}, S_{l}^{*}, I_{l}^{*}, T_{l}^{*}, S_{m}^{*}, I_{m}^{*}, T_{p}^{*}\right)$, be the equilibrium point of the system (1). Then, setting the right hand side of system (1) to zero, we obtain

$$
\left\{\begin{array}{l}
\pi_{h}-\lambda_{h m} S_{h}-\left(\mu_{h}+\delta_{h}\right) S_{h}+\eta_{h} T_{h}=0, \\
\lambda_{h m} S_{h}-\left(\mu_{h}+\delta_{h}+\gamma_{h}\right) I_{h}=0, \\
\gamma_{h} I_{h}-\left(\mu_{h}+\eta_{h}+\delta_{h}\right) T_{h}=0, \\
\pi_{l}-\lambda_{l m} S_{l}-\mu_{l} S_{l}+\eta_{l} T_{l}=0, \\
\lambda_{l m} S_{l}-\left(\mu_{l}+\delta_{l}+\gamma_{l}\right) I_{l}=0, \\
\gamma_{l} I_{l}-\left(\eta_{l}+\delta_{l}+\mu_{l}\right) T_{l}=0, \\
\pi_{m}-\left(\lambda_{m l}+\lambda_{m h}\right) S_{m}-\left(\mu_{m}+\sigma_{m}\right) S_{m}=0 \\
\left(\lambda_{m l}+\lambda_{m h}\right) S_{m}-\left(\mu_{m}+\gamma_{m}\right) I_{m}=0, \\
\rho T_{h}-\left(\mu_{h}+\delta_{h}\right) R_{h}=0, \\
\sigma_{m} S_{m}+\gamma_{m} I_{m}=0 .
\end{array}\right.
$$

From the second, fourth and sixth equations of (4), we write $I_{h}^{*}$ and $I_{l}^{*}$ in terms of $I_{m}^{*}$. From the sixth equation of (4) we have,

$$
\frac{\beta_{2} \beta_{3}\left(\mu_{l}+\delta_{l}+\gamma_{l}\right) I_{m}^{*} S_{m}^{*}+\beta_{1} \beta_{4}\left(\mu_{h}+\delta_{h}+\gamma_{h}\right) I_{m}^{*} S_{m}^{*}}{\left(\mu_{h}+\delta_{h}+\gamma_{h}\right)\left(\mu_{l}+\delta_{l}+\gamma_{l}\right) N_{m}^{*}}-\left(\mu_{m}+\sigma_{m}\right) I_{m}=0
$$

defining

$$
\mathcal{R}_{0}=\frac{\beta_{2} \beta_{3}\left(\mu_{l}+\delta_{l}+\gamma_{l}\right)+\beta_{1} \beta_{4}\left(\mu_{h}+\delta_{h}+\gamma_{h}\right)}{\left(\mu_{h}+\delta_{h}+\gamma_{h}\right)\left(\mu_{l}+\delta_{l}+\gamma_{l}\right)} .
$$

Equation (2) reduces to (3).

\subsection{Disease Free Equilibrium}

This solution $I_{h}^{*}=I_{l}^{*}=I_{m}^{*}=0$ of 4 leads to the disease-free equilibrium point $E_{0}$ is given by

$$
E_{2}=\left(\frac{\pi_{h}}{\mu_{h}}, 0,0, \frac{\pi_{l}}{\mu_{l}}, 0,0, \frac{\pi_{m}}{\mu_{m}}, 0,0,0\right) .
$$

\subsection{The Effective Reproductive Number, $\mathcal{R}_{\text {eff }}$}

In this section, the threshold parameter that governs the spread of a disease referred to as the effective reproduction number is determined. Mathematically, it is the spectral radius of the next generation matrix [10]. The equations of the system (1) are re-written starting with infective classes, to obtain 


$$
\left\{\begin{array}{l}
S_{h}^{\prime}=\pi_{h}-\lambda_{h m} S_{h}-\left(\mu_{h}+\delta_{h}\right) S_{h}+\eta_{h} T_{h} \\
I_{h}^{\prime}=\lambda_{h m} S_{h}-\left(\mu_{h}+\delta_{h}+\gamma_{h}\right) I_{h}, \\
T_{h}^{\prime}=\gamma_{h} I_{h}-\left(\mu_{h}+\eta_{h}+\delta_{h}\right) T_{h}, \\
S_{l}^{\prime}=\pi_{l}-\lambda_{l m} S_{l}-\mu_{l} S_{l}+\eta_{l} T_{l}, \\
I_{l}^{\prime}=\lambda_{l m} S_{l}-\left(\mu_{l}+\delta_{l}+\gamma_{l}\right) I_{l}, \\
T_{l}^{\prime}=\gamma_{l} I_{l}-\left(\eta_{l}+\delta_{l}+\mu_{l}\right) T_{l}, \\
S_{m}^{\prime}=\pi_{m}-\left(\lambda_{m l}+\lambda_{m h}\right) S_{m}-\left(\mu_{m}+\sigma_{m}\right) S_{m} \\
I_{m}^{\prime}=\left(\lambda_{m l}+\lambda_{m h}\right) S_{m}-\left(\mu_{m}+\gamma_{m}\right) I_{m}, \\
R_{h}^{\prime}=\rho T_{h}-\left(\mu_{h}+\delta_{h}\right) R_{h}, \\
T_{p}^{\prime}=\sigma_{m} S_{m}+\gamma_{m} I_{m} .
\end{array}\right.
$$

From the system (6), $\mathcal{F}_{i}$ and $\mathcal{V}_{i}$ are defined as

$$
\mathcal{F}_{i}=\left[\begin{array}{c}
\frac{\beta_{1} I_{m}}{N_{h}} S_{h} \\
\frac{\beta_{2} I_{m}}{N_{l}} S_{l} \\
\left(\frac{\beta_{3} I_{h}}{N_{m}}+\frac{\beta_{4} I_{l}}{N_{m}}\right) S_{m}
\end{array}\right], \mathcal{V}_{i}=\left[\begin{array}{c}
\left.\left(\mu_{h}+\delta_{h}+\gamma_{h}\right)\right) I_{h} \\
\left(\mu_{l}+\delta_{l}+\gamma_{l}\right) I_{l} \\
\left(\mu_{m}+\delta_{m}+\gamma_{m}\right) I_{m}
\end{array}\right]
$$

substituting $I_{h}=\frac{\beta_{1} I_{m}}{\mu_{h}+\delta_{h}+\gamma_{h}}$ and $I_{l}=\frac{\beta_{2} I_{m}}{\mu_{l}+\delta_{l}+\gamma_{l}}, \mathcal{F}_{i}$ becomes,

$$
\mathcal{F}_{i}=\left[\begin{array}{c}
\beta_{1} I_{m} \\
\beta_{2} I_{m} \\
\left(\frac{\beta_{2} \beta_{3} I_{m}}{\mu_{l}+\delta_{l}+\gamma_{l}}+\frac{\beta_{1} \beta_{4} I_{m}}{\mu_{h}+\delta_{h}+\gamma_{h}}\right) I_{m}
\end{array}\right] .
$$

The partial derivatives of $\mathcal{F}_{i}$ and $\mathcal{V}_{i}$ with respect to $I_{h}, I_{l}$ and $I_{m}$ and evaluating at the disease free point gives

$$
F=\left[\begin{array}{ccc}
0 & 0 & \beta_{1} \\
0 & 0 & \beta_{2} \\
0 & 0 & \left(\frac{\beta_{2} \beta_{3} I_{m}}{\mu_{l}+\delta_{l}+\gamma_{l}}+\frac{\beta_{1} \beta_{4}}{\mu_{l}+\delta_{l}+\gamma_{l}}\right)
\end{array}\right], V=\left(\begin{array}{ccc}
\left(\mu_{h}+\delta_{h}+\gamma_{h}\right) & 0 & 0 \\
0 & \left(\mu_{l}+\delta_{l}+\gamma_{l}\right) & 0 \\
0 & 0 & \left(\mu_{m}+\gamma_{m}\right)
\end{array}\right] .
$$

$F V^{-1}$ is computed and obtained as

$$
F V^{-1}=\left[\begin{array}{ccc}
0 & 0 & \frac{\beta_{1}}{\mu_{m}+\gamma_{m}} \\
0 & 0 & \frac{\beta_{2}}{\mu_{m}+\gamma_{m}} \\
0 & 0 & \left(\frac{\beta_{2} \beta_{3} I_{m}}{\mu_{l}+\delta_{l}+\gamma_{l}}+\frac{\beta_{1} \beta_{4}}{\mu_{l}+\delta_{l}+\gamma_{l}}\right) \frac{1}{\mu_{m}+\gamma_{m}}
\end{array}\right] .
$$


The eigenvalues of $F V^{-1}$ are $\lambda_{1}=\lambda_{2}=0$ and

$$
\lambda_{3}=\frac{\beta_{2} \beta_{3}\left(\mu_{l}+\delta_{l}+\gamma_{l}\right)+\beta_{1} \beta_{4}\left(\mu_{h}+\delta_{h}+\gamma_{h}\right)}{\left(\mu_{m}+\gamma_{m}\right)\left(\mu_{h}+\delta_{h}+\gamma_{h}\right)\left(\mu_{l}+\delta_{l}+\gamma_{l}\right)} .
$$

The effective reproduction number $\mathcal{R}_{\text {eff }}$ measures the average number of new infections generated by a typical infectious individual in a community when treatment and trapping strategies are in place. As we increase trapping and treatment rates, $\beta_{i}$ have the effect of increasing $\mathcal{R}_{\text {eff }}$ because of linearity of $\mathcal{R}_{\text {eff }}$ in terms of $\beta_{i}$ taking into account that, treatment and trapping are effective.

\section{Local Stability of the Disease Free-Equilibrium}

The disease-free equilibrium point is $E_{2}=\left(\frac{\pi_{h}}{\mu_{h}}, 0,0, \frac{\pi_{l}}{\mu_{l}}, 0,0, \frac{\pi_{m}}{\mu_{m}}, 0,0,0\right)$ Thus, the Jacobian matrix $J_{E 2}$ of the system (1) is computed by differentiating each equation in the system with respect to the state variables $S_{h}, I_{h}, T_{h}, S_{l}, I_{l}, T_{l}, S_{m}, I_{m}, T_{p}$. Hence, at the steady states the Jacobian matrix for system (1) is given by

$$
J_{E_{0}}=\left[\begin{array}{cccccccccc}
-\mu_{h} & 0 & 0 & \eta_{h} & 0 & 0 & 0 & 0 & -\beta_{1} & 0 \\
0 & -b & 0 & 0 & 0 & 0 & 0 & 0 & \beta_{1} & 0 \\
0 & \rho_{h} & -\mu_{h} & 0 & 0 & 0 & 0 & 0 & 0 & 0 \\
0 & \gamma_{h} & 0 & -d & 0 & 0 & 0 & 0 & 0 & 0 \\
0 & 0 & 0 & 0 & -\mu_{l} & 0 & \eta_{l} & 0 & -\beta_{2} & 0 \\
0 & 0 & 0 & 0 & 0 & -a & \eta_{l} & 0 & \beta_{2} & 0 \\
0 & 0 & 0 & 0 & 0 & \gamma_{l} & -q & 0 & \beta_{2} & 0 \\
0 & -\beta_{4} & 0 & 0 & 0 & -\beta_{3} & 0 & -s & 0 & 0 \\
0 & \beta_{4} & 0 & 0 & 0 & \beta_{3} & 0 & 0 & -\left(\mu_{m}+\gamma_{m}\right) & 0 \\
0 & 0 & 0 & 0 & 0 & 0 & 0 & \sigma_{m} & \gamma_{m} & -\mu_{m}
\end{array}\right]
$$

where $a=\mu_{l}+\delta_{l}+\gamma_{l}, \quad d=\mu_{h}+\eta_{h}, \quad q=\mu_{l}+\delta_{l}+\eta_{l}$ and $r=\mu_{m}+\gamma_{m}$ The characteristic polynomial is given as

$$
\lambda^{2}+\lambda\left(\mu_{h}+\delta_{h}+\mu_{m}\right)+\mu_{h} \beta_{4}+\mu_{h} \mu_{m}+\delta_{h} \beta_{4}+\delta_{h} \mu_{m}-\beta_{3} \beta_{3}=0
$$

Using Birkhoff and Rota's theorem on the differential inequality (3) we obtain

$$
\left|J_{E_{0}}-\lambda\right|=0 \text {. }
$$

From the matrix (7) we note that the first, third, fourth, fifth and sixth have diagonal entries. Therefore their corresponding eigenvalues are;

$$
\begin{aligned}
\lambda_{1} & =\mu_{h}, \lambda_{3}=-\mu_{h}, \lambda_{4}=-d, \lambda_{5}=-\mu_{l}, \lambda_{8}=-s, \lambda_{1}=-\mu_{m}, \\
J_{E_{0}} & =\left[\begin{array}{cccc}
-b & 0 & 0 & \beta_{1} \\
0 & -a & \eta_{l} & \beta_{2} \\
0 & \gamma_{l} & -q & 0 \\
\beta_{4} & \beta_{3} & 0 & -r
\end{array}\right] .
\end{aligned}
$$

With the help of mathematical software, the following characteristic equation is obtained

$$
\begin{aligned}
& \lambda^{2}\left(\beta_{2} \beta_{3}+\beta_{4} \beta_{1}\right)-\lambda\left[(b+q) \beta_{2} \beta_{3}+(r+q) a+\gamma_{l} \eta_{l}+(a-q) \beta_{1} \beta_{4}\right]+b q \beta_{2} \beta_{3}+r \gamma_{l} \eta_{l}+a q \beta_{4} \beta_{1} \gamma_{l} \eta_{l}, \\
& \lambda^{2}\left(\beta_{2} \beta_{3}+\beta_{4} \beta_{1}\right)-\lambda\left[(b+q) \beta_{2} \beta_{3}+(r+q) a+\gamma_{l} \eta_{l}+(a ? q) \beta_{1} \beta_{4}\right]+b q \beta_{2} \beta_{3}+r \gamma_{l} \eta_{l}+a q \beta_{4} \beta_{1} \gamma_{l} \beta_{l} \\
& \lambda^{2}\left(\beta_{2} \beta_{3}+a+\beta_{4} \beta_{1}\right)-\lambda\left[(b+q) \beta_{2} \beta_{3}+(r+q) a+\gamma_{l} \eta_{l}+(a ? q) \beta_{1} \beta_{4}\right]+b q \beta_{2} \beta_{3}+r \gamma_{l} \eta_{l}+a q \beta_{4} \beta_{1} \gamma_{l} \beta_{l}=0
\end{aligned}
$$

and 


$$
\lambda_{3}=\frac{\beta_{2} \beta_{3}\left(\mu_{l}+\delta_{l}+\gamma_{l}\right)+\beta_{1} \beta_{4}\left(\mu_{h}+\delta_{h}+\gamma_{h}\right)}{\left(\mu_{m}+\gamma_{m}\right)\left(\mu_{h}+\delta_{h}+\gamma_{h}\right)\left(\mu_{l}+\delta_{l}+\gamma_{l}\right)}-\left(\mu_{m}+\gamma_{m}\right)=\left(\mu_{m}+\gamma_{m}\right)\left(\mathcal{R}_{\text {eff }}-1\right) .
$$

If $\mathcal{R}_{\text {eff }}<1$, then $\lambda_{1}, \lambda_{2}, \lambda_{1}, \lambda_{3}, \lambda_{4}, \lambda_{5}, \lambda_{6}, \lambda_{7}, \lambda_{8}, \lambda_{9}$ and $\lambda_{10}$ are all negative. These results are summarised with the following theorem

\section{Theorem}

The disease-free equilibrium point is locally asymptotically stable if $\mathcal{R}_{\text {eff }}<1$ and unstable if $\mathcal{R}_{\text {eff }}>1$.

\section{The Endemic Equilibrium, $E_{3}$}

In the presence $s$ of infection, that is, $I_{h}^{*} \neq 0, I_{l}^{*} \neq 0 I_{h}^{*} \neq 0$, the model system (1) has a non-trivial equilibrium point, $E_{3}$ called the endemic equilibrium point which is given by $E_{3}=\left(S_{h}^{*}, I_{h}^{*}, T_{h}^{*}, S_{l}^{*}, I_{l}^{*}, T_{l}^{*} S_{m}^{*}, I_{m}^{*}, T_{m}^{*}, T_{p}^{*}\right) \neq 0$, where $S_{h}^{*}, I_{h}^{*}, T_{h}^{*}, S_{l}^{*}, I_{l}^{*}, T_{l}^{*} S_{m}^{*}, I_{m}^{*}, T_{m}^{*}, T_{p}^{*}>0$ from the system (4.3). In this case, the following solution is considered

$$
\mathcal{R}_{0} S_{m}^{*}=N_{m}^{*}
$$

where $\mathcal{R}_{\text {eff }}$ is derived above. Then from the equations of system (4.3) we obtain

$$
\begin{aligned}
S_{h}^{*} & =\frac{\pi_{h}^{2}}{\left(\pi_{h}+\beta_{1} I_{m}^{*}\right) \mu_{h}} \\
S_{l}^{*} & =\frac{\pi_{l}^{2}}{\left(\pi_{l}+\beta_{2} I_{m}^{*}\right) \mu_{l}} \\
I_{h}^{*} & =\frac{\beta_{1} I_{m}^{*} \pi_{h}}{\left(\pi_{h}+\beta_{1} I_{m}^{*}\right)\left(\mu_{h}+\delta_{h}+\gamma_{h}\right)} \\
T_{l}^{*} & =\frac{\gamma_{l} \beta_{2} I_{m}^{*}}{\left(\mu_{l}+\delta_{l}+\gamma_{l}\right)\left(\mu_{l}+\eta_{l}\right)} \\
I_{l}^{*} & =\frac{\beta_{1} I_{m}^{*} \pi_{l}}{\left(\pi_{l}+\beta_{1} I_{m}^{*}\right)\left(\mu_{l}+\delta_{l}+\gamma_{l}\right)} \\
T_{h}^{*} & =\frac{\gamma_{h} \beta_{2} I_{h}}{\left(\mu_{h}+\delta_{h}+\gamma_{h}\right)\left(\mu_{h}+\eta_{h}\right)} .
\end{aligned}
$$

We let $d=\mu_{l}+\delta_{l}+\gamma_{l}$ and $l=\mu_{h}+\delta_{h}+\gamma_{h}$ then

$$
S_{m}^{*}=\frac{\pi_{m} l d\left(\pi_{l}+\beta_{2} I_{m}^{*}\right)\left(\pi_{h}+\beta_{1} I_{m}^{*}\right)}{\left(\beta_{1} I_{m}^{*} \pi_{h}\right) l \beta_{2} \beta_{4} \pi_{l}+\left(\pi_{l}+\beta_{2} I_{m}^{*}\right) d \beta_{1} \beta_{3} \pi_{l}}
$$

Adding the last two equations of the system and making some simplifications we obtain

$$
B I_{m}^{2}+B_{1} I_{m}+A=0
$$

where

$$
\begin{aligned}
B= & \beta_{1} \beta_{2} \pi_{m}\left(\mu_{l}+\delta_{l}+\gamma_{l}\right)\left(\mu_{h}+\delta_{h}+\gamma_{h}\right)+\left(\mu_{m}+\gamma_{m}\right) \\
B_{1}= & \pi_{m}\left(\beta_{2} \pi_{h}+\beta_{1} \pi_{l}\right)\left(\mu_{h}+\delta_{h}+\gamma_{h}\right)\left(\mu_{l}+\delta_{l}+\gamma_{l}\right)+\left(\mu_{m}+\gamma_{m}\right) \beta_{1} \beta_{2} \pi_{h} \pi_{l}\left(\mu_{l}+\delta_{l}+\gamma_{l}\right) \\
& -\beta_{1} \beta_{2} \pi_{h} \pi_{l}\left(\mu_{l}+\delta_{l}+\gamma_{l}\right)-\beta_{1} \beta_{2} \beta_{4} \pi_{l}^{2} \pi_{l}\left(\mu_{h}+\delta_{h}+\gamma_{h}\right) \\
A= & \pi_{m} \pi_{l} \pi_{h}\left(\mu_{h}+\delta_{h}+\gamma_{h}\right)\left(\mu_{l}+\delta_{l}+\gamma_{l}\right)\left(1-\mathcal{R}_{\text {eff }}\right) .
\end{aligned}
$$

The equation, $f\left(I_{m}^{*}\right)=0$ corresponds to a situation when the disease persists (endemic). In case of backward bifurcation, multiple endemic equilibrium must exist. This implies that while considering the equation 
(4.18) there are three cases we have to consider depending on the signs of $B_{1}$ and $A$ since $B$ is always positive. That is;

1) If $B_{1}<0$ and $A=0$ or $B_{1}^{2}-4 B A=0$, then Equation (4.18) has a unique endemic equilibrium point (one positive root) and there is no possibility of backward bifurcation.

2) If $A>0, B_{1}>0$ and $B_{1}^{2}-4 B A>0$, then Equation (4.18) has two endemic equilibria (two positive roots), and thus there is the possibility of backward bifurcation to occur.

3) Otherwise, there is none.

However it is important to note that $\mathrm{A}$ is always positive if $\mathcal{R}_{\text {eff }}<1$ and negative if $\mathcal{R}_{\text {eff }}>1$. Hence the above explanation leads to the following theorem.

Theorem 5 The rift valley fever basic model has,

1) Precisely one unique endemic equilibrium if $A<0 \Leftrightarrow \mathcal{R}_{\text {eff }}>1$

2) Precisely one unique endemic equilibrium if $B_{1}<0$ and $A=0$ or $B_{1}^{2}-4 B A=0$

3) Precisely two endemic equilibrium if $A>0, B_{1}<0$ and $B_{1}^{2}-4 B A>0$

4) None, otherwise.

From (iii) it is observed that backward bifurcation is possible if the discriminant is set $B_{1}^{2}-4 B A=0$ and solve for the critical value of $\mathcal{R}_{\text {eff }}$. Thus, we get

$$
\mathcal{R}_{\text {eff }}^{c}=1-\frac{B^{2}}{4 A \pi_{h} \pi_{l} \pi_{m}\left(\mu_{h}+\delta_{h}+\gamma_{h}\right)\left(\mu_{l}+\delta_{l}+\gamma_{l}\right)}
$$

where backward bifurcation occurs for values of $\mathcal{R}_{\text {eff }}$ lying in the range $\mathcal{R}_{0}^{c}<\mathcal{R}_{0}<1$. The theorem below gives the condition of existence of the endemic equilibrium point, $E_{3}$.

Theorem 5 The endemic equilibrium point, $E_{3}$ exists if $\mathcal{R}_{0}>1$.

\section{Sensitivity Analysis}

Sensitivity analysis determines parameters that have a high impact on $\mathcal{R}_{\text {eff }}$ and should be targeted by intervention strategies. We will use the approach done in [11] and Blower and Dowlatabadi, 1994 to calculate the sensitivity indices of the effective reproduction number, $\mathcal{R}_{\text {eff }}$.

The indices are crucial and will help us determine the importance of each individual parameter in transmission dynamics and prevalence of the Rift Valley Fever Virus.

Definition 1 The normalized forward sensitivity index of a variable, $u$, that depends differentiably on index on a parameter, $p$ is defined as; $\Upsilon_{p}^{u}=\frac{\partial u}{\partial p} \times \frac{p}{u}$.

The analytical expression for the sensitivity of $\mathcal{R}_{\text {eff }}$ is $\Upsilon^{\mathcal{R}_{\text {eff }}}=\frac{\partial u}{\partial p} \times \frac{p}{\mathcal{R}_{\text {eff }}}$ for each of the parameter $p$ involved in $\mathcal{R}_{\text {eff }}$. We used the following parameter values to determine the sensitivity indices; $\beta_{1}=6.91$, $\beta_{2}=0.31, \beta_{3}=0.31, \beta_{4}=0.31, \mu_{h}=0.20, \mu_{l}=0.20, \mu_{m}=0.20, \delta_{h}=0.50, \delta_{l}=0.50, \gamma_{h}=0.50, \gamma_{l}=0.6, \sigma_{m}=0.20$

\section{Interpretation of Sensitivity Analysis}

From Table 1, it shows that when the parameters $\beta_{1}, \beta_{2}, \beta_{3}, \beta_{4}, \mu_{h}, \mu_{l}$, and $\mu_{m}$, are increased keeping other parameters constant they increase the value of $\mathcal{R}_{\text {eff }}$ implying that they increase the the burden of the disease among the human, animals and vector populations as they have positive indices. While the parameters $\sigma_{m}$, $\gamma_{h}, \gamma_{l}$ and $\gamma_{m}$ decrease the value of $\mathcal{R}_{\text {eff }}$ when they are increased while keeping the other parameters constant, implying that they decrease the burden of the disease among the human, livestock and vector populations. The specific interpretation of each parameter shows that, the most sensitive parameter is the transmission rates for susceptible cattle individuals with infection $\beta_{2}$ followed by $\beta_{1}$ transmission rates for susceptible human individuals with infection and so on as the Table 1 indicates.

\section{Numerical Simulation}

We carry out numerical simulations for mathematical model of rift valley fever for the set of parameters from literature as shown in Table 1 . The parameter values that changed the value of $\mathcal{R}_{e 0}$ are: $\beta_{1}=2.9 ; \beta_{2}=2.9$; 
$\beta_{3}=0.531 ; \delta_{h}=0.5 ; \pi_{h}=100000, \pi_{l}=100000, \pi_{h}=100000$, and $\delta_{l}=0.3$.

We have the following simulation results (Figures 1-6). Figure 1 shows variation of the different populations for specified parameter values. As treatment rates $T_{h}$ and $T_{l}$ increase, both infected human population $I_{h}$ and cattle population $I_{l}$ rises quickly to reach maximum and then drops to a steady state. Corresponding to the rise of both human population $I_{h}$ and livestock population $I_{l}$ infective there is a drop in the susceptible human $S_{h}$ and livestock $S_{l}$ population until reaches the minimum values and then rises to a steady state. The reduction of mosquitoes $S_{l}$ and $I_{m}$ through trapping $T_{p}$ of both infected and susceptible lead to reduction in infected human and animal population because the two are infected by infected mosquitoes and they do not infect each other. The simulation results depicted in Figure 2 illustrating the the endemic state with the value of $\mathcal{R}_{\text {eff }}=1.2804$. The results show the introduction of trapping the mosquitoes, treating human and livestock populations reduce the reproduction number from 8.60276 to 0.4782 , this implies the clearance of the disease.

Table 1. Parameter values and the calculated sensitivity indices.

\begin{tabular}{ccc}
\hline Parameter symbol & Value & Sensitivity Index \\
\hline$\pi_{m}$ & 100,000 & -0.0000023 \\
$\pi_{l}$ & 100,000 & -0.000139643 \\
$\mu_{m}$ & 0.8 & 0.108334 \\
$\gamma_{m}$ & 0.9 & 0.131592 \\
$\gamma_{h}$ & 0.5 & -0.186422 \\
$\beta_{2}$ & 2.9 & 0.133382 \\
$\gamma_{l}$ & 0.5 & -0.215213 \\
$\pi_{h}$ & 100,000 & 0.322104 \\
$\mu_{l}$ & 0.2 & -1.191842 \\
$\mu_{h}$ & 0.2 & -1.191842 \\
$\delta_{l}$ & 0.5 & -1.191842 \\
$\delta_{h}$ & 0.5 & -1.191842 \\
$\beta_{1}$ & 0.531 & 2.48506
\end{tabular}

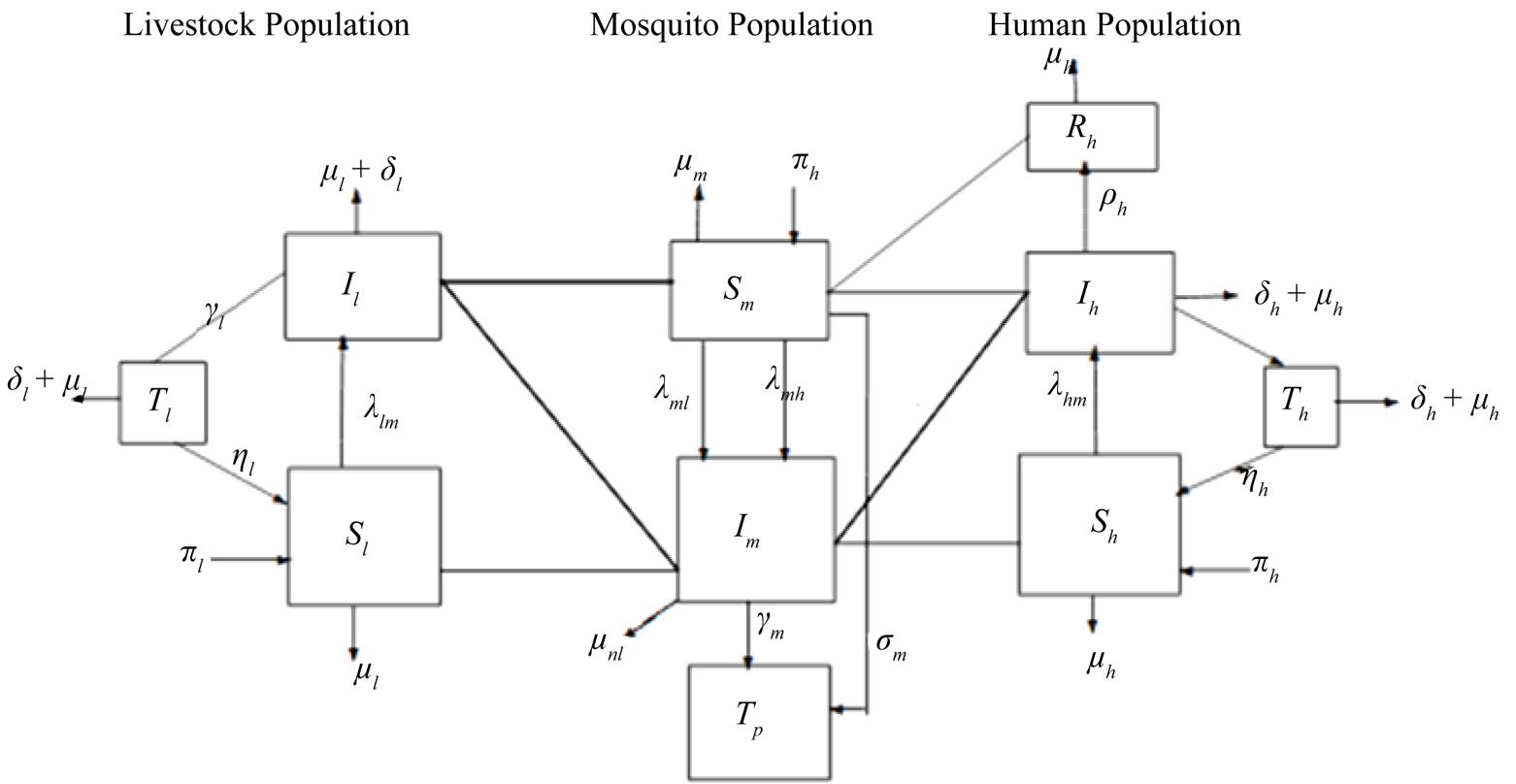

Figure 1. Schematic diagram for Rift Valley Fever Model with interventions. 

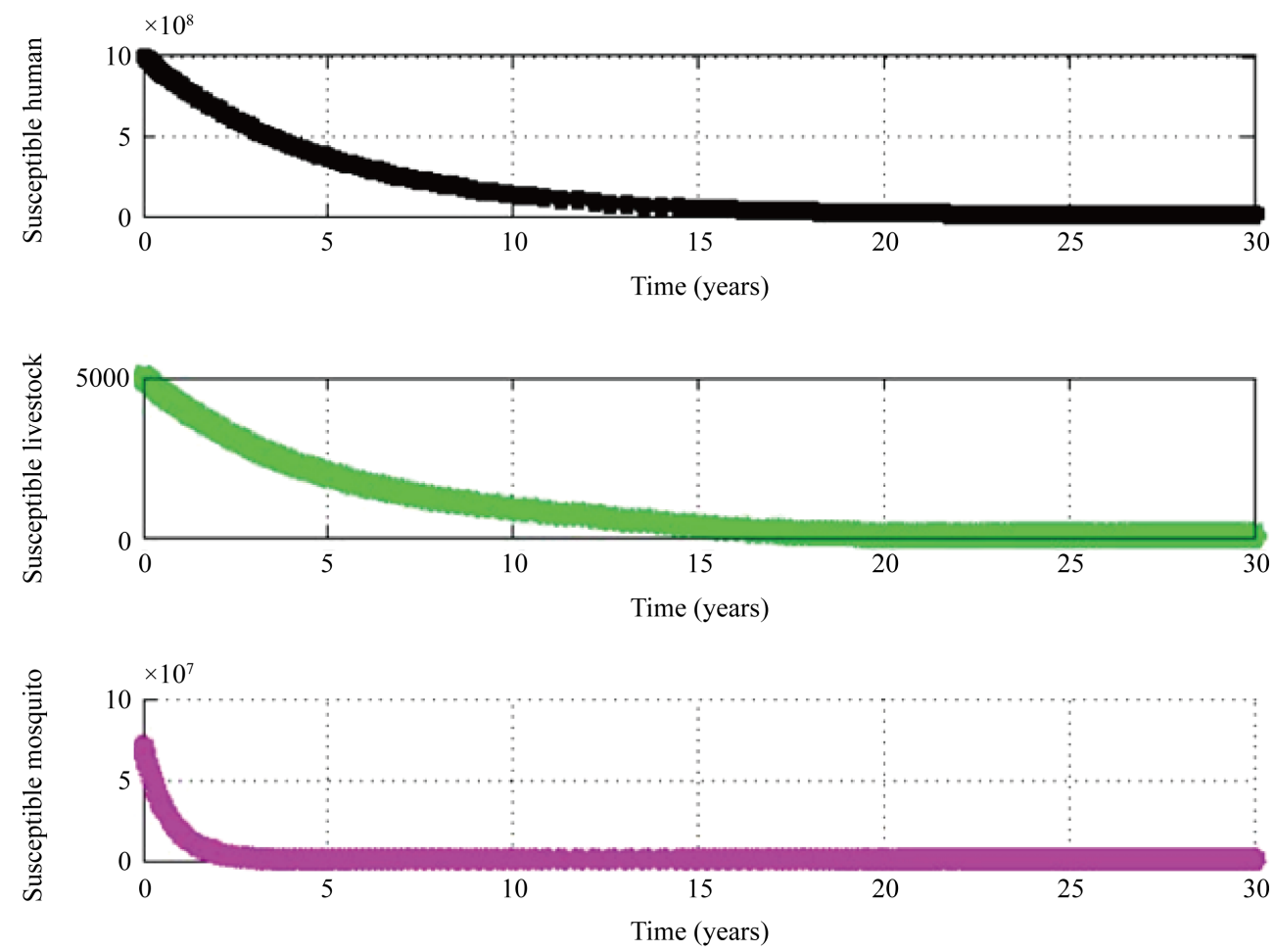

Figure 2. Population Dynamics of the rift valley fever without intervention model.

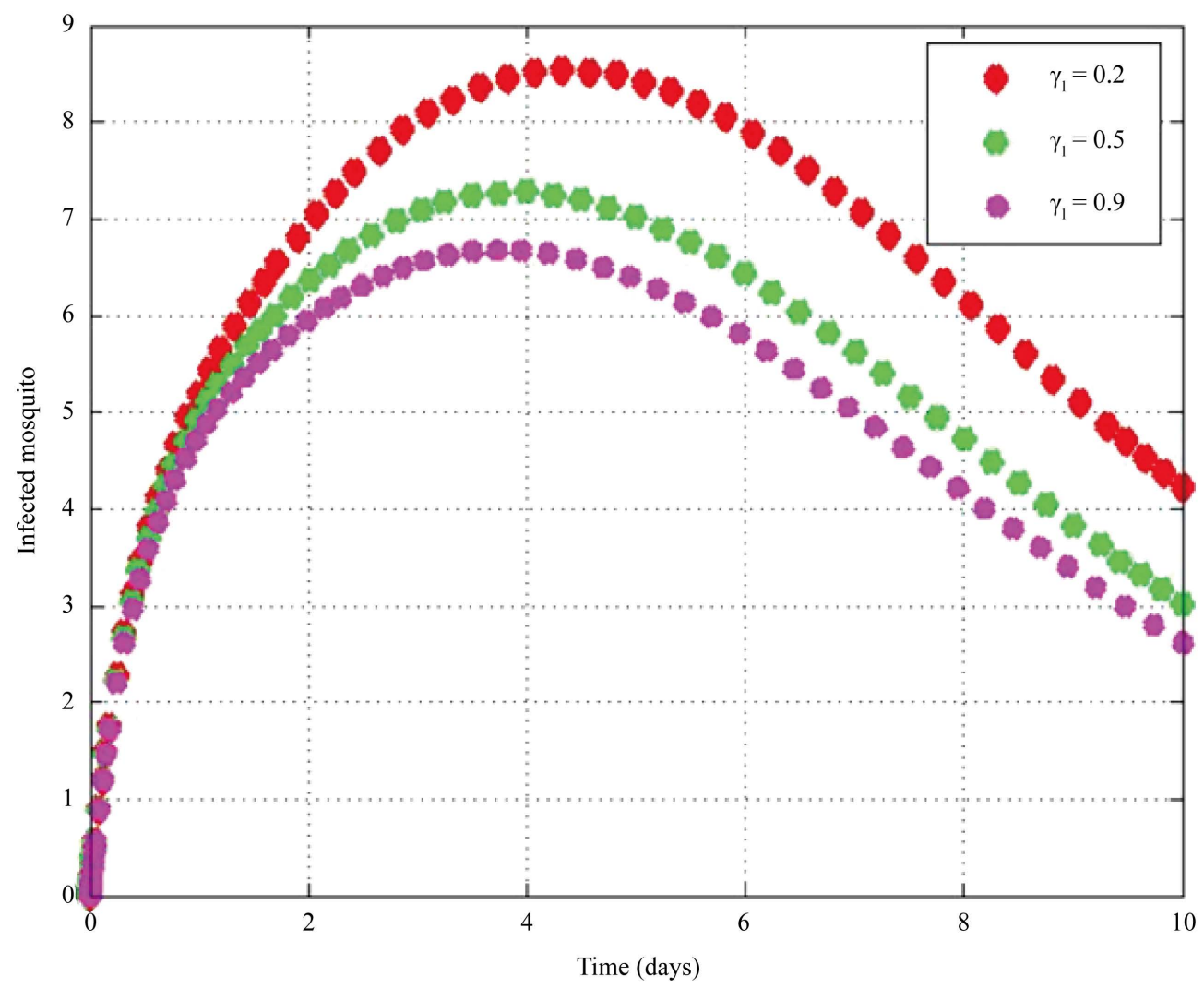

Figure 3. Effects of treatment of livestock on mosquitoes population. 


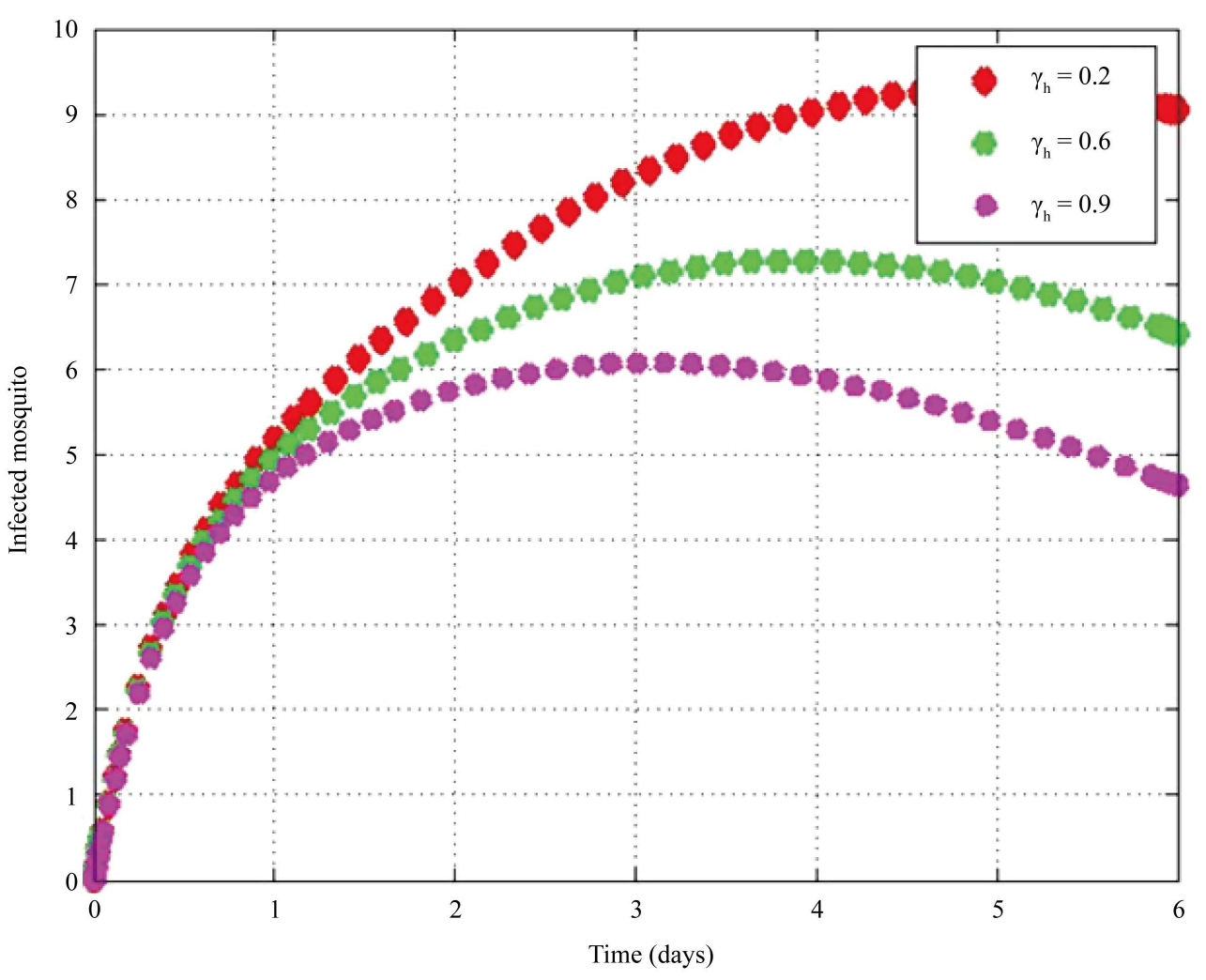

Figure 4. Effects of treatment of human on mosquitoes population.

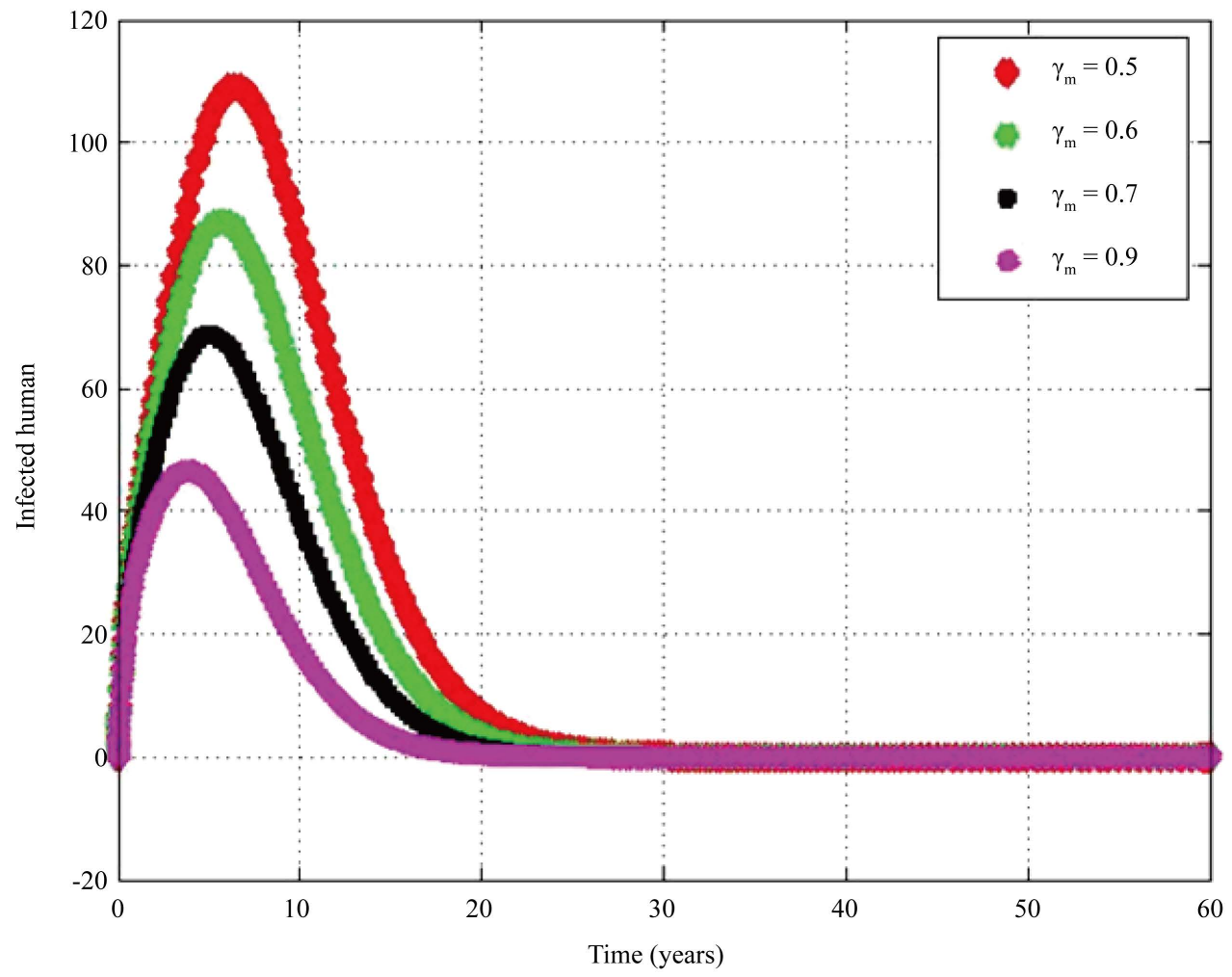

Figure 5. Effects of trapping of mosquitoes on human population. 


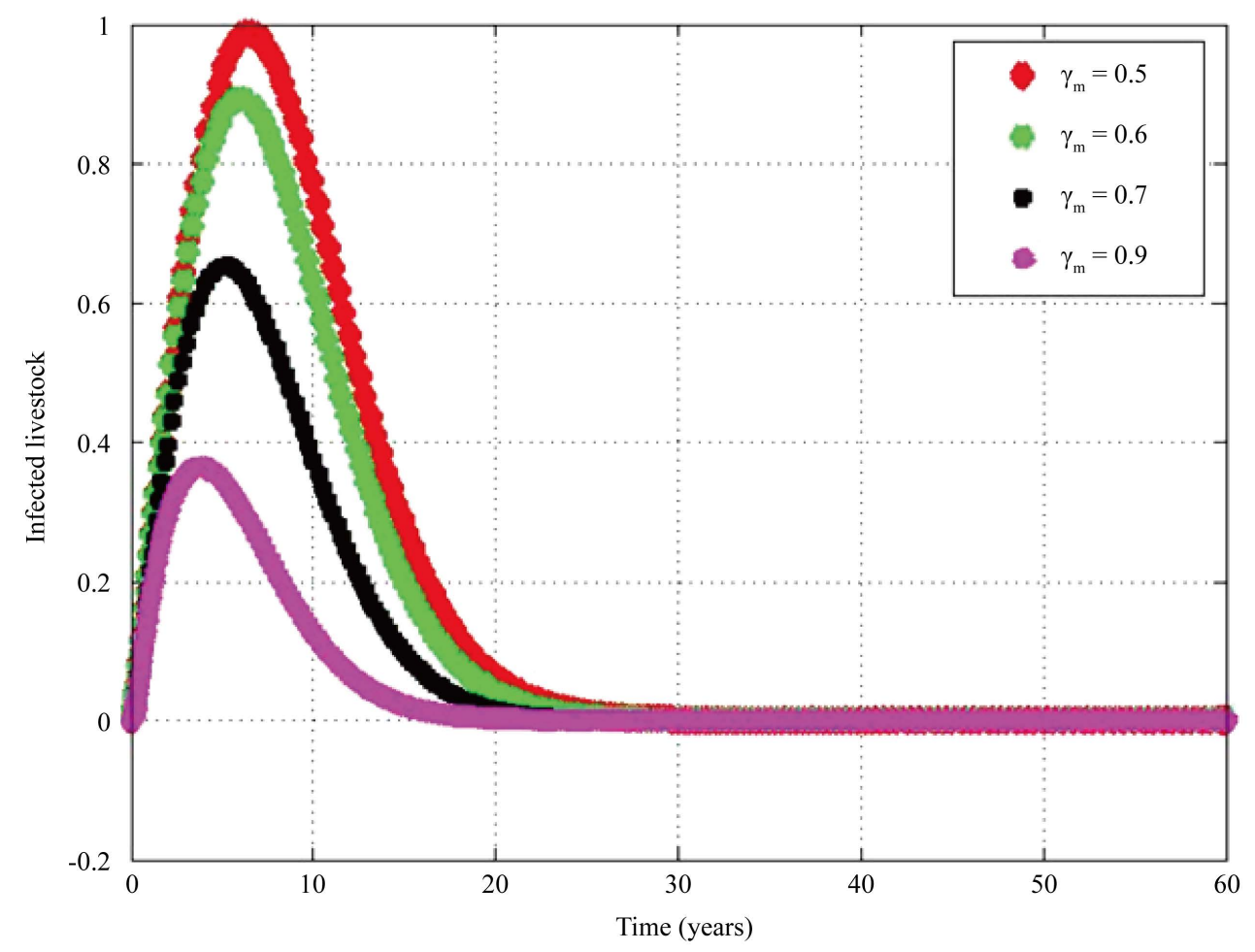

Figure 6. Effects of trapping of mosquitoes on human population.

\subsection{Variation of Different Parameters on the Dynamics of Rift Valley Fever Model with Treatment and Trapping}

In this section parameters, $\gamma_{h}, \gamma_{l}$, and $\sigma_{p}$ representing the treatment rate for the infected human population, treatment rate for the infected livestock population and the trapping rate for the mosquitoes population respectively were varied to determine their effect on the different model populations. When the treatment rates of livestock and human increase the infected human, livestock and mosquitoes decrease as the Figure depicts. When the trapping rate $\sigma_{m}$ and $\gamma_{m}$ of the infected and susceptible mosquitoes respectively increase, the infected human and livestock decrease. This implies that endemicity of the disease among human and livestock decreases.

\subsection{Discussion}

The Rift Valley Model formulated in this study is well posed and exists in a feasible region where disease free and endemic equilibrium points are obtained and their stability investigated. The model has two interventions; treatment for human and livestock and trapping for mosquitoes. We have shown that disease free equilibrium exist and is locally asymptotically stable whenever its associated effective reproduction number $\mathcal{R}_{\text {eff }}$ is less than unity, and it has a unique endemic equilibrium u when $\mathcal{R}_{\text {eff }}$ exceeds unity. These results have important public health implications, since they determine the severity and outcome of the epidemic (i.e. clearance or persistence of infection) and provide a framework for the design of control strategies. Analysis of the model show that in the absence of treatment of livestock and human and trapping of mosquitoes (ie. $\sigma_{m}=\gamma_{h}=\gamma_{L}=0$ ) and if $\mathcal{R}_{\text {eff }}>1$, the epidemic will develop,but if $\mathcal{R}_{\text {eff }}<1$ it will die out. At $\gamma_{h}=\gamma_{l}=\infty$ (all infected human and cattle have access to treatment for human and all mosquitoes are trapped), then $\mathcal{R}_{\text {eff }}=0$, and the epidemic will be fully controlled. The main epidemiological findings of this study include:

- In the absence of treatment of human or livestock and trapping for mosquitoes: $\mathcal{R}_{\text {eff }}>1$ implying that treatment failure leads the epidemic persistence. Hence the combination of treatment for livestock, humans and trapping for mosquitoes can eradicate the rift valley fever infection if $\mathcal{R}_{\text {eff }}$ can be reduced to below unity. 
- With human or livestock and trapping for mosquitoes $\mathcal{R}_{\text {eff }}<1$, so; (a) $\mathcal{R}_{\text {eff }}<1$ : human or livestock treatment and trapping for mosquitoes is effective, hence elimination of infection.

\section{Conclusion}

In this paper, the rift valley fever model with interventions was formulated and analysed. Using the theory of differential equations, the invariant set in which the solutions of the model are biologically meaningful was derived. Boundedness of solutions was also proved. Analysis of the model showed that there exist two possible solutions, namely the disease-free point and the endemic equilibrium point. Further analysis showed that the disease-free point is locally stable implying that small perturbations and fluctuations on the disease state will always result in the clearance disease if $\mathcal{R}_{\text {eff }}<1$. In the final analysis treatment and trapping interventions program will effectively control the spread of rift valley fever.

\section{References}

[1] Pepin, M., Bouloy, M., Bird, M., Kempand, B.H. and Paweska, A. (2010) Rift Valley Fever Virus (Bunyaviridae: Phlebovirus): An Update on Pathogenesis, Molecular Epidemiology, Vectors, Diagnostics and Prevention. Veterinary Research, 41, 61. http://dx.doi.org/10.1051/vetres/2010033

[2] Gaff, H.D., Hartley, D.M and Leahy, N.P. (2007) An Epidemiological Model of Rift Valley Fever. Electronic Journal of Di-Erential Equations, 1, 12.

[3] Clements, A.C., Pfeifer, D.U., Martin, V. and Otte, M.J. (2007) A Rift Valley Fever Atlas for Africa. Preventive Veterinary Medicine, 2, 72-78. http://dx.doi.org/10.1016/j.prevetmed.2007.05.006

[4] Fischer, E., Boender, G.J. and De Koeijer, A.A. (2013) The Transmission Potential of Rift Valley Fever Virus among Livestock in the Netherlands: A Modeling Study. Veterinary Research, 44, 58. http://dx.doi.org/10.1186/1297-9716-44-58

[5] Métras, R., Collins, L.M., White, R.G., Alonso, S. and Chevalier, V. (2011) Rift Valley Fever Epidemiology, Surveillance, and Control: What Have Models Contributed? Vector Borne and Zoonotic Diseases, 11, 761-771. http://dx.doi.org/10.1089/vbz.2010.0200

[6] Mpeshe, S.C., Haario, H. and Tchuenche, J.M. (2011) A Mathematical Model of Rift Valley Fever with Human Host. Acta Biotheoretica, 59, 231-250. http://dx.doi.org/10.1007/s10441-011-9132-2

[7] Niu, T.C., Gaff, H.D., and Papelis, Y.E. and Hartley, D.M. (2012) An Epidemiological Model of Rift Valley Fever with Spatial Dynamics. Computational and Mathematical Methods in Medicine, 2012, Article ID: 138757.

[8] Chitnis, N., James, M.H. and Carrie, A.M. (2013) Modelling Vertical Transmission in Vectorborne Diseases with Applications to Rift Valley Fever. Journal of Biological Dynamics, 7, 11-40. http://dx.doi.org/10.1080/17513758.2012.733427

[9] Otte, J.M., Nugent, R. and McLeod, A., Eds. (2004) Transboundary Animal Diseases: Assessment of Socio-Economic Impacts and Institutional Response. Volume 9, FAO, Livestock Policy Discussion Paper 9. http://www.fao.org/ag/againfo/resources/en/publications/

[10] Van Den, D.P. and Watmough, J. (2002) Reproduction Numbers and Sub-Threshold Endemic Equilibria for Compartmental Models of Disease Transmission. Mathematical Biosciences, 80, 29. http://dx.doi.org/10.1016/S0025-5564(02)00108-6

[11] Chitnis, N., Hyman, J.M. and Cushing, J.M. (2008) Determining Important Parameters in the Spread of Malaria through the Sensitivity Analysis of a Mathematical Model. Bulletin of Mathematical Biology, 70, 1272-1296. http://dx.doi.org/10.1007/s11538-008-9299-0 